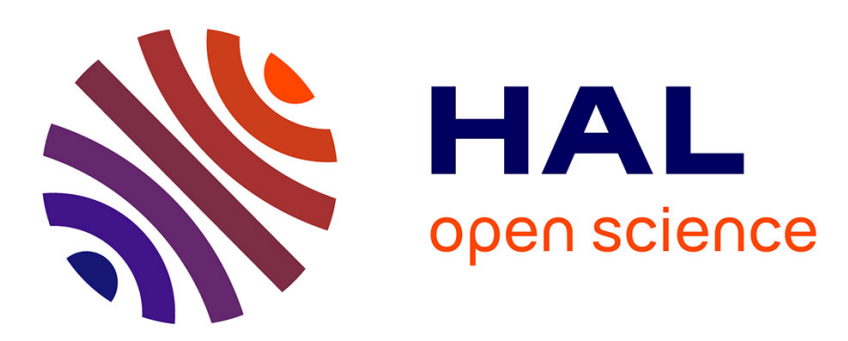

\title{
Surfaces sélectives : propriétés optiques et estimation de l'efficacité énergétique dans l'application aux récepteurs solaires
}

\author{
G. Gouhman, M. Koudrachova, N. Milevskaya, F. Eydinova
}

\section{- To cite this version:}

G. Gouhman, M. Koudrachova, N. Milevskaya, F. Eydinova. Surfaces sélectives : propriétés optiques et estimation de l'efficacité énergétique dans l'application aux récepteurs solaires. Revue de Physique Appliquée, 1980, 15 (3), pp.393-396. 10.1051/rphysap:01980001503039300 jpa-00244742

HAL Id: jpa-00244742

https://hal.science/jpa-00244742

Submitted on 1 Jan 1980

HAL is a multi-disciplinary open access archive for the deposit and dissemination of scientific research documents, whether they are published or not. The documents may come from teaching and research institutions in France or abroad, or from public or private research centers.
L'archive ouverte pluridisciplinaire HAL, est destinée au dépôt et à la diffusion de documents scientifiques de niveau recherche, publiés ou non, émanant des établissements d'enseignement et de recherche français ou étrangers, des laboratoires publics ou privés. 


\title{
Surfaces sélectives : propriétés optiques et estimation de l'efficacité énergétique dans l'application aux récepteurs solaires
}

\author{
G. Gouhman, M. Koudrachova, N. Milevskaya et F. Eydinova \\ Institut d'énergétique de G. M. Krjijanovsky
}

(Reçu le 16 novembre 1979, accepté le 19 novembre 1979)

\begin{abstract}
Résumé. - On étudie le problème d'utilisation des revêtements sélectifs sur les surfaces planes des récepteurs solaires.

On examine les types principaux des revêtements avec leurs caractéristiques optiques, techniques et d'exploitation.

On analyse les données théoriques et expérimentales concernant l'influence des surfaces sélectives sur l'efficacité des récepteurs solaires plans.
\end{abstract}

\begin{abstract}
The problem of use of selective coatings on the flat surfaces of solar receivers is studied. The main types of coatings together with optical, technical and exploitation characteristics are examined. Finally, theoretical and experimental data concerning the influence of selective surfaces on the efficiency of flat solar receivers are discussed.
\end{abstract}

Les revêtements sélectifs appliqués aux surfaces des récepteurs solaires augmentent réellement leur rendement. On distingue deux classes de revêtements.

La première comprend les revêtements ayant un pouvoir d'absorption élevé pour le rayonnement solaire $\left(\alpha_{S}\right)$ et un pouvoir émissif faible pour le rayonnement propre de la surface $(\varepsilon)$ à la température de travail $\left(T_{\gamma}\right)$.

La deuxième classe est composée de revêtements transparents aux rayonnements solaires, qui sont capables de réfléchir le rayonnement thermique provenant de la surface chauffée du capteur.

Seuls les revêtements de la première classe sont l'objet de la présente étude.

La surface d'un récepteur idéal aurait un coefficient de réflexion nul pour le rayonnement solaire, et un coefficient de réflexion égal à l'unité pour le rayonnement infrarouge (IR).

Un exemple de surface sélective de ce type a été mis au point à l'Institut de Kyijanovsky. Sur une surface de cuivre sont revêtues trois couches. Les paramètres optiques deviennent :

$$
\alpha_{\mathrm{S}}=0,93 \text { et } \varepsilon=0,06
$$

Le coefficient de réflexion de cette surface en jonction de la longueur d'onde est représenté par la figure 1 (Réf. [1]).

Des courbes semblables caractérisent le revêtement obtenu par un procédé de technologie différente (Réf. [2]).

Afin que la sélectivité de la surface se traduise par une absorption maximale dans le spectre solaire et minimale en infrarouge (pour l'intervalle correspondant à la température de travail), le changement de valeur du coefficient de réflexion doit s'effectuer pour une valeur optimale $\lambda_{0}$ de la longueur d'onde, la situation idéale étant :

$$
\begin{gathered}
R_{\lambda}=0 \text { pour } 0<\lambda<\lambda_{0} \\
R+1 \text { pour } \lambda_{0}<\lambda<\infty .
\end{gathered}
$$

La valeur limite $\lambda_{0}$ est bien déterminée par l'intersection de deux courbes :

- l'une qui décrit la distribution énergétique du rayonnement solaire et l'autre

- l'énergie de rayonnement propre de la surface.

Par conséquent $\lambda_{0}$ dépend de $T_{\mathrm{r}}$ (et du degré de concentration du rayonnement solaire, lorsqu'il s'agit d'un capteur focalisant).

Si $T_{\mathrm{r}}$ augmente (et $\eta$ aussi) $\lambda_{0}$ diminue.

Il convient de définir l'efficacité de la surface de réception $\eta_{\mathrm{s}}$, comme le rapport entre la quantité d'énergie solaire captée et reçue par cette surface.

On considère la radiation comme seul moyen d'échanges thermiques de la surface avec le milieu ambiant. 
L'expression du $\eta_{\mathrm{s}}$ s'obtient à partir du bilan thermique de la surface, pour la température $T_{\mathrm{r}}$, et les propriétés optiques définies par $\alpha_{\mathrm{s}}$ et $\varepsilon$, il vient :

$$
\begin{aligned}
\eta_{\mathrm{s}}\left(\alpha_{\mathrm{s}}, \varepsilon,\right. & \left.T_{\mathrm{r}}\right)= \\
= & \frac{n S\left(1-\gamma_{\mathrm{v}}-\alpha_{\mathrm{v}}\right) \alpha_{\mathrm{s}}-\varepsilon \sigma\left(T_{\mathrm{r}}^{4}-T_{\alpha}^{4}\right)\left(1-\gamma_{\mathrm{v} 1}-\alpha_{\mathrm{v} 1}\right)}{n S}
\end{aligned}
$$

où

$S$ : flux solaire incident,

$\alpha_{s}$ : moyenne intégrale de valeurs du coefficient d'absorption pour le rayonnement solaire,

$$
\varepsilon \text { : moyenne, }
$$

dans l'intervalle $\lambda_{0}<\lambda<\infty$.

$\gamma_{\mathrm{v}}$ et $\alpha_{\mathrm{v}}$ : valeurs moyennes des coefficients de réflexion et d'absorption du verre pour $0<\lambda<\lambda_{0}$;

$\gamma_{\mathrm{v} 1}$ et $\alpha_{\mathrm{v} 1}$ : mêmes paramètres pour $\lambda_{0}<\lambda<\infty$.

$n$-degré de concentration du rayonnement. On s'aperçoit que l'effet du revêtement sélectif sur la surface du récepteur plan $(n=1)$ devient plus sensible avec l'augmentation de la température de cette surface. Les pertes de chaleur provoquées par rayonnement croissant proportionnellement à $T_{\mathrm{r}}^{4}$. Le rendement du récepteur baisse. Cependant, l'utilisation de revêtements sélectifs permet à cette baisse d'être beaucoup moins significative [3]. Dans le cadre du développement des recherches sur l'énergie solaire, on étudie des méthodes très diverses pour obtenir des surfaces sélectives.

On peut sélectionner quelques groupes de surfaces sélectives, partant des principes physiques qui sont à leur origine.

I $^{\text {er }}$ groupe. - Oxydes noirs de métaux déposés sur la surface polie d'un métal ayant un pouvoir de réflexion élevé. La valeur élevée de $\alpha_{s}$ pour le rayonnement solaire et la transparence simultanée aux rayons $\lambda>\lambda_{0}$ sont assurés par le choix de l'épaisseur optimale de la couche de métal oxydé.

La faible valeur du $\varepsilon$ est liée au pouvoir de réflexion de la surface métallique. Il existe plusieurs techniques pour obtenir les couches d'oxydes noirs à la surface d'un métal :

a) Oxydation thermique du métal à l'air ou à l'oxygène. A titre d'exemple citons le cuivre recouvert par une couche de son oxyde dont les caractéristiques optiques sont les suivantes :

$$
\alpha_{\mathrm{s}}=0,62 \div 0,8 \quad \varepsilon=0,2 \quad[4] ;
$$

b) Attaque chimique de la surface d'un métal, par exemple : oxydation du cuivre

$$
\alpha_{\mathrm{s}}=0,95 \quad \varepsilon=0,11 \quad[5] ;
$$

c) Pulvérisation d'une solution moussante sur la surface chauffée d'un métal; par exemple : l'oxyde cuivrique sur de l'aluminium

$$
\alpha_{\mathrm{s}}=0,93 ; \quad \varepsilon=0,11 \quad[6] ;
$$

d) Evaporation sous vide des métaux ou évaporation des oxydes métalliques sous vide élevé.

Par exemple :

$\rightarrow$ évaporation de l'or pour obtenir de l'or noir [7];

$\rightarrow$ revêtement de $\mathrm{Cr}_{2} \mathrm{O}_{3}$ sur du chrome avec $\alpha_{\mathrm{s}}=0,92$ et $\varepsilon=0,05$ obtenu sous vide élevé [8].

e) Electrolyse.

Exemple : oxyde cobaltique ou alliage chromenickel-vanadium sur du nickel poli [9].

Ce groupe contient également les revêtements du type :

nickel noir $\left(\alpha_{\mathrm{s}}=0,95, \varepsilon=0,07\right)[10]$ et

chrome noir $\left(\alpha_{\mathrm{s}}=0,92-0,96, \varepsilon=0,08 \div 0,12\right)[11]$.

II $^{\mathbf{e}}$ groupe. - Peinture sélective composée de petites particules de semi-conducteur réparties dans un composant organique.

Le film de peinture est déposé sur la surface métallique dont le pouvoir de réflexion est élevé. On choisit le semi-conducteur opaque pour $\lambda<\lambda_{0}$ et transparent pour $\lambda>\lambda_{0}$.

La sélectivité de la peinture augmente grâce à l'augmentation de $\alpha_{\mathrm{s}}$ dans l'intervalle $\lambda<\lambda_{0}$, due à la diffusion et des réflexions multiples sur les particules du semi-conducteur dont la taille est comparable avec la longueur d'onde $\lambda_{0}$.

Afin d'obtenir une faible valeur de $\varepsilon$, le composant organique utilisé doit être transparent aux rayons infrarouges. L'exemple de ce revêtement est le film de silicium et caoutchouc organique dans lequel sont réparties les particules de $\mathrm{PbS}$. Ce revêtement a des caractéristiques suivantes :

$$
\alpha_{\mathrm{s}}=0,85 ; \quad \varepsilon=0,13-0,16 \quad[12] .
$$

Les couches minces de peinture et d'émail noirs appartiennent également à ce groupe. La seule différence par rapport aux revêtements décrits ci-dessus est que les particules du semi-conducteur sont remplacées par les particules du pigment noir. Leurs paramètres caractéristiques sont les suivants :

$$
\alpha_{\mathrm{s}}=0,95 \div 0,97, \quad \varepsilon=0,2 \div 0,4 \text {. }
$$

Pour améliorer ces caractéristiques, il faut utiliser des substances organiques bien transparentes qui ne changent pas de propriétés sous l'action de l'ultraviolet.

III ${ }^{\mathrm{e}}$ groupe. - Surface sélective formée par un film de semi-conducteur déposé sur du métal poli.

Le semi-conducteur choisi doit vérifier la condition : l'extrémité de sa bande d'absorption coïncide avec $\lambda_{0}$. Ce type de revêtement représente par exemple le film de silicium monocristal en sandwich avec une couche d'aluminium [13].

La valeur caractéristique $\alpha_{\mathrm{s}}=0,65(\varepsilon=0,12)$ est faible à cause de la réflexion intense à la surface du semi-conducteur, étant donné la valeur élevée de son 
indice de réfraction. On peut diminuer la réflection au moyen de revêtements spéciaux - antireflet sur la surface du semi-conducteur. Cela complique la technologie et augmente le coût de production.

Les revêtements sont employés pour des températures très élevées.

IV e groupe. - Surface formée par une couche d'un semi-conducteur dopé.

Pour le rayonnement infrarouge, le pouvoir de réflexion de cette surface approche le pouvoir de réflexion du métal sous-jacent.

Pour $\lambda>\lambda_{0}$, le pouvoir de réflexion est déterminé par le coefficient de réfraction.

Les paramètres caractéristiques sont les suivants :

$$
\alpha=0,94, \quad \varepsilon=0,2 \div 32 \text {. }
$$

Les revêtements de ce groupe s'appliquent également aux hautes températures.

$V^{e}$ groupe. - Revêtements multi-couches antireflet sur la surface polie du métal.

Le fonctionnement de ces revêtements est basé sur le principe de l'interférométrie dans les couches minces. On choisit comme matière soit un semi-conducteur, soit un diélectrique dont les caractéristiques optiques sont bien déterminées. L'épaisseur de chaque couche doit être égale au multiple entier de la quantité $\lambda_{\max } / 4$, où $\lambda_{\max }$ désigne la longueur d'onde qui correspond au maximum d'intensité lumineuse du spectre solaire. Ces revêtements sont transparents au rayonnement infrarouge, ce qui garantit la valeur faible du $\varepsilon$. Comme exemple, on peut citer le revêtement à trois couches :

$$
\mathrm{Al}_{2} \mathrm{O}_{3}-\mathrm{M}_{0}-\mathrm{Al}_{2} \mathrm{O}_{3}
$$

déposé sur du molybdène $(\alpha=0,83, \varepsilon=0,05)$ [14] et le revêtement étudié à l'E.N.I.N. :

$$
\mathrm{Ni}-\mathrm{ZnS}-\mathrm{MgF}_{2}\left(\alpha_{\mathrm{s}}=0,92 ; \varepsilon=0,07\right) \quad[1]
$$

Ce dernier peut être chauffé jusqu'à $150^{\circ} \mathrm{C}$ à l'air et il a subi avec succès les essais dans les conditions naturelles du sud de l'U.R.S.S.

La technique utilisée pour créer ce type de revêtement ne se prête pas aux températures trop élevées.

On estime la qualité de la surface sélective par la valeur du rappórt; $\alpha_{\mathrm{s}} / \varepsilon$. Plus cette valeur est élevée, plus la sélectivité de la surface devient bonne.

Si les échanges thermiques entre la surface sélective et le milieu ambiant sont dus à la radiation seule, la température du récepteur solaire est déterminée par les rapports $\alpha_{\mathrm{s}} / \varepsilon$.

Dans le cas où une importante quantité de chaleur, absorbée par le récepteur est évacuée par l'intermédiaire d'un vecteur de transfert thermique, et si les pertes thermiques sont dues à la convection ou à la conduction (comme, par exemple, pour les chauffe- eau solaires), il faut tenir compte des valeurs de $\alpha_{\text {s }}$ et $\varepsilon$ séparément.

Le critère pour le choix d'un type de revêtement sélectif, en vue de son application aux centrales solaires, n'est pas basé uniquement sur la valeur des paramètres optiques. Il faut tenir compte d'autres paramètres importants, à savoir :

- résistance et solidité des revêtements pour de longues périodes d'exploitation,

- technologie de leur fabrication,

- possibilité d'utilisation à grande échelle,

- coût.

Des revêtements étudiés très récemment possèdent des propriétés optiques voisines des caractéristiques optimales, mais ils ne sont pas suffisamment résistants pendant l'exploitation.

L'électrolyse chimique (déposition et oxydation des métaux) est la méthode la plus accessible pour fabriquer des revêtements sélectifs de la façon industrielle. Ainsi, on peut obtenir les revêtements de grande surface des récepteurs solaires plans.

Les revêtements, tels que le chrome noir sur de l'acier [11] et le nickel noir, ce dernier composé de deux couches [10], ont de bonnes caractéristiques optiques. Ils résistent aux variations de température, mais l'action de l'humidité les détruit.

On a mis au point un procédé peu coûteux de fabrication des revêtements sélectifs par oxydation en faisant traiter des feuilles de cuivre dans plusieurs bains chimiques. Cette méthode attire l'attention par sa simplicité et la possibilité de sa réalisation dans des conditions industrielles. Les paramètres optiques des revêtements ainsi obtenus $\left(\alpha_{\mathrm{s}}=0,95, \varepsilon=0,12-0,13\right)$ s'avèrent suffisamment bons pour leur application aux capteurs solaires plans, où la valeur de $\alpha_{s}$ doit être élevée. Cependant, ces revêtements ne résistent pas à des températures élevées et à l'humidité.

Le coût des revêtements obtenus par la méthode de pulvérisation n'est pas élevée. Cette technologie est simple, mais la reproduction infidèle des propriétés optiques, la nécessité de polir au préalable la surface du métal et de contrôler avec précision l'épaisseur de la couche du revêtement diminuent le mérite de cette méthode.

Pour l'estimation de l'efficacité énergétique des revêtements sélectifs appliqués aux récepteurs solaires plans, il convient de préciser les intervalles de température pour lesquels on préfère une surface noire dont le coefficient $\alpha_{\mathrm{s}}$ est très élevé.

L'analyse des données quantitatives concernant l'influence de l'application des revêtements sélectifs $[4,15,16]$ nous conduit aux conclusions suivantes : l'application des revêtements sélectifs augmente l'efficacité du récepteur solaire plan lorsque la température de sa surface atteint $60^{\circ}-70^{\circ} \mathrm{C}$ pour des températures plus basses il est plus rentable d'utiliser la surface noire, car les déperditions thermiques dues à l'émissivité sont encore négligeables et le pouvoir 


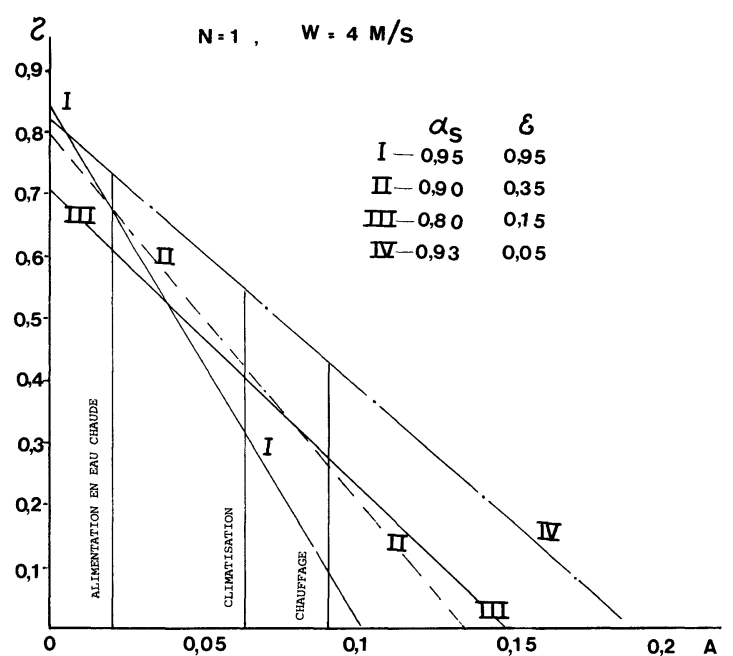

Fig. 1.

d'absorption de la surface noire est supérieur à $\alpha_{s}$ des surfaces sélectives.

Pour les mêmes raisons, on choisit plutôt le revêtement dont $\alpha_{\mathrm{s}}$ est élevé que celui dont la valeur du rapport $a_{\mathrm{s}} / \varepsilon$ est élevée.

Cette affirmation est appuyée par les graphiques (Figs. 1 et 2) qui représentent l'efficacité du récepteur solaire plan $(\eta)$ en fonction de l'ensemble des paramètres décrivant ses conditions de travail

$$
\left(A=\frac{T_{\mathrm{r}}-T_{\mathrm{A}}}{S}\right) \text {. }
$$

Les calculs ont été faits pour les récepteurs ayant des propriétés optiques différentes.

Pour les conditions de fonctionnement des récepteurs le plus souvent rencontrées :

— période d'été $\left(S=700 \mathrm{~W} / \mathrm{m}^{2}\right)$; alimentation en eau chaude $\left(T_{\mathrm{r}}=40^{\circ} \mathrm{C}\right.$ et $\left.T_{\mathrm{a}}=30^{\circ} \mathrm{C}\right)$ et climatisation $\left(T_{\mathrm{r}}=75^{\circ} \mathrm{C}, T_{\mathrm{a}}=30^{\circ} \mathrm{C}\right)$;

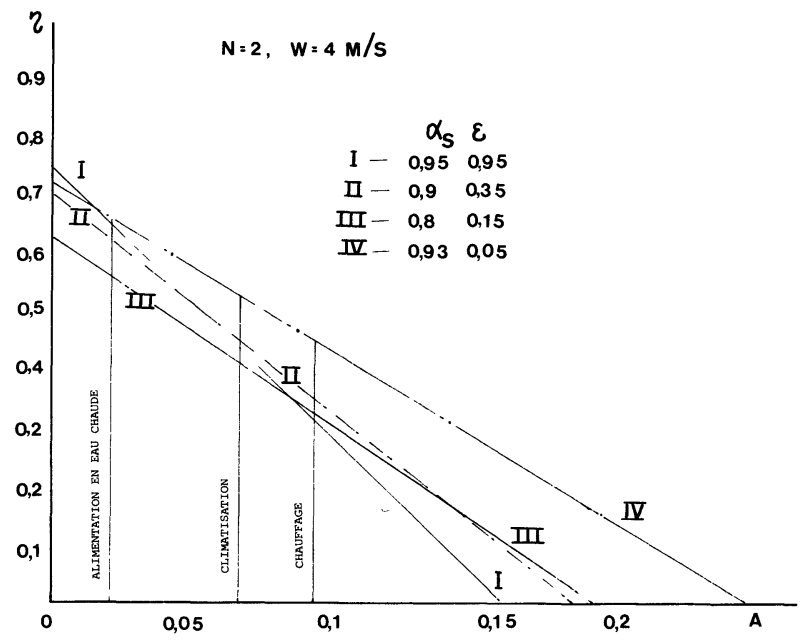

Fig. 2.

- période d'hiver $\left(S=500 \mathrm{~W} / \mathrm{m}^{2}\right)$ chauffage $\left(T_{\mathrm{r}}=60^{\circ} \mathrm{C}, T_{\mathrm{a}}=15^{\circ} \mathrm{C}\right)$.

On constate que les revêtements caractérisés par $\alpha_{\mathrm{s}}=0,8$ et $\varepsilon=0,15\left(\alpha_{\mathrm{s}} / \varepsilon=5,3\right)$ soit moins rentables que les revêtements pour lesquels $\alpha_{s}=0,9$ et $\varepsilon=0,35\left(\alpha_{\mathrm{s}} / \varepsilon=2,6\right)$ et, dans certaines conditions, moins rentables que les surfaces noires.

L'étude économique des revêtements sélectifs est entreprise dans la publication [16]. On examine un modèle expérimental de chauffe-eau solaires dont la surface de réception est recouverte par des revêtements sélectifs élaborés par l'E.N.I.N. $\left(\alpha_{\mathrm{s}}=0,89-0,9\right)$.

L'étude expérimentale et le calcul montre que l'utilisation des revêtements sélectifs augmente le rendement du chauffe-eau solaire à partir de $T_{\mathrm{r}}=50^{\circ} \mathrm{C}$.

Mais du point de vue économique la rentabilité n'est admise qu'à partir de $T_{\mathrm{r}} \geqslant 60^{\circ} \mathrm{C}$. Pour $T_{\mathrm{r}}=70^{\circ} \mathrm{C}$ l'efficacité du chauffe-eau à surface sélective est de 1,8 fois supérieure à celle du chauffe-eau à surface noire.

\section{Bibliographie}

[1] Koudriachova, M. D., Nouveaux revêtements sélectifs pour les surfaces de capteurs, Héliotechnique, 1969, No 4.

[4] Gue-Syn-CHI, Etudes des propriétés de surfaces noires à absorption sélective.

[5] KelleR, Traitement chimique des surfaces pour l'obtention de revêtements sélectifs, Congrès Int. à Paris, Le soleil au service de l'homme, 1973.
[15] Garf, B. A., Vladimirova, L. N., Changement des caractéristiques techniques de la centrale solaire du type piège à calories dû à l'utilisation des surfaces sélectives, Héliotechnique, 1970, $\mathrm{n}^{\circ} 5$, p. 35-41.

[16] Bahoum, V. A., BieckTeniev, M. B., Influence des revêtements sélectifs sur le bilan thermique du récepteur solaire (Jzviesta AN T.S.S.R.), FTH et G.N., $\mathrm{n}^{\circ} 1,1970$. 